\title{
Wissenschaftspolitische Herausforderungen für die Informatik
}

\author{
Gregor Snelting \\ Universität Karlsruhe \\ Lehrstuhl Programmierparadigmen \\ snelting@ipd.info.uni-karlsruhe.de
}

\begin{abstract}
Zusamenfassung. Der folgende Beitrag - in der ursprünglichen Form vorgetragen auf dem Jahrestreffen 2008 des Beirates der Universitätsprofessoren der Gesellschaft für Informatik (GIBU) behauptet, dass im Gefolge des Bologna-Prozesses und der Exzellenzinitiative das Niveau der Ausbildung in technischen Fächern, insbesondere in der Informatik, im Durchschnitt sinken wird. Da Deutschland sich dies nicht leisten kann, andererseits Bologna unumkehrbar ist, werden Kompensationsmaßnahmen diskutiert.
\end{abstract}

Wie kann das Informatikstudium im Gefolge von Bologna und Exzellenzinitiative für alle Absolventen ein international konkurrenzfähiges Ausbildungsniveau sichern?

\section{Hintergrund}

Deutschland lebt von seiner technischen Kompetenz. Wir sind Exportweltmeister, weil wir die besten Autos und Maschinen bauen, zusammen mit der geballten Informatik, die heute in technischen Systemen notwendig ist. Unsere technische Kompetenz ist deshalb unersetzliche Grundlage unserer ökonomischen Existenz. Die Öffentlichkeit und die Politik verstehen aber nicht ausreichend, dass technische Fächer eine spezifische Fachkultur und spezifische Erfordernisse in Lehre und Forschung haben.

Ich behaupte: Dieser mangelnde Respekt vor den Besonderheiten technischer Fächer hat im Gefolge des Bologna-Prozesses und der Exzellenziniative negative Auswirkungen auf das durchschnittliche Niveau der Ausbildung; mit nachteiligen Folgen für die internationale Konkurrenzfähigkeit Deutschlands. Die GI muss deshalb, zusammen mit 4ING und TU9, darüber nachdenken, wie man diese negativen Auswirkungen kompensiert. Dabei sieht sich der GIBU als einschlägiges GI-Gremium besonders in der Pflicht, die Belange der Forschung und der wissenschaftsorientierten Ausbildung zu vertreten.

Einige Anregungen dazu möchte ich in diesem Beitrag geben. Einige der Punkte, die ich ansprechen möchte, wurden kürzlich auch in einem Positionspapier von BITKOM, GI und 4ING zusammengefasst [1]. Man muss aber, wie ich finde, noch deutlicher und noch subtiler argumentieren, und vor allem über konkrete Maßnahmen reden.

\section{Bachelor/Master vs Diplom}

Ich behaupte also, dass die Umstellung auf Bachelor/Master zusammen mit der Exzellenziniative gerade in den technischen Fächern negative Auswirkungen auf die Konkurrenzfähigkeit Deutschlands hat, jedenfalls wenn es bei der bisherigen Implementierung bleibt. Um dies zu verstehen, werfen wir einen Blick zurück auf die fast ausgestorbene Welt des Diploms. Nicht nur ich bin überzeugt, dass das Rückgrat unserer technischen Stärke in den Diplom-Studiengängen lag. Denn das Unversitätsstudium eines Ingenieurfaches war in Deutschland immer wesentlich anspruchsvoller als in anderen Ländern. 
Anders als z.B. im angelsächsischen Raum, wurden vom Beginn des Studiums an wissenschaftliche Grundlagen und Mathematik intensiv vermittelt. Tatsächlich kam Berufspraktisches erst spät im Studium vor, was von vielen auch kritisiert wurde. Ich behaupte aber: diese Studiums-Struktur war im internationalen Vergleich ein Standortvorteil. Denn sie bot talentierten Studenten optimale Entwicklungsmöglichkeiten. Der Grund ist, dass man im Alter um die 20 am intelligentesten und lernfähigsten ist, und diese Zeit muss man nutzen, um die jungen Talente optimal zu fördern und zu fordern. Diese Talente sind es nämlich, die später durch Kompetenz und Innovationen den Exportüberschuss generieren. Das hat im alten System gut funktioniert, und deshalb ist der Dipl.-Ing. ein internationales Markenzeichen. Selbstverständlich kann man darüber reden, was denn nun die wirklich wichtigen wissenschaftlichen Grundlagen sind; das ändert nichts an meiner Überzeugung, dass sie früh vermittelt werden sollten.

Übrigens ist mir völlig klar, dass im alten System keineswegs alle Studenten den wissenschaftlichen Grundlagen gewachsen waren. Diese Tatsache war ja auch ein Motiv für das neue Bachelor/Master System. Aber ich bleibe dabei: für talentierte Studenten bot das Diplomstudium optimale Entwicklungsmöglichkeiten und nutzte die Lernfähigkeit optimal aus. Wissenschaftliche Grundlagen oder Mathematik später nachzuholen ist viel schwieriger. Und eins muss klar sein: so wichtig „praxisorientierte“ Absolventen sind, so wichtig sind auch wissenschaftliche Grundlagen, wenn wir international mithalten wollen. Denn praxisorientierte Absolventen auf Bachelor-Niveau kann man auch in anderen Ländern günstig bekommen.

Im neuen Bachelor/Master System findet sich genau das umgekehrte Modell zum alten Diplom. Der Bachelor muss primär berufsqualifizierend sein, die wissenschaftliche Vertiefung soll im Master stattfinden. Gleichzeitig will die Politik die Zahl der Master-Studenten deckeln und allenfalls die Hälfte der Bachelor-Absolventen zum Master zulassen. Die Politik verspricht sich davon eine Durchsatzerhöhung: mehr Hochschulabsolventen bei reduzierten Kosten und Abbrecherquoten. Dieses Hoffnung mag für manche Studienfächer richtig sein; im Falle technischer Fächer führt das Bachelor/Master System aber - wie ich noch zeigen werde - zu einer Reduktion der durchschnittlichen Qualifikation, die wir uns nicht leisten können.

Angesichts der ökonomischen Bedeutung von Technik war deshalb eine erste einmütige Reaktion der Ingenieursfächer auf Bologna die Aussage, dass natürlich der Master mindestens das Niveau des Diploms in Breite und Tiefe haben muss, und dass wir in Deutschland nicht weniger technische Master brauchen, sondern mehr. Sowohl TU9 als auch das neue BITKOM/GI Papier haben dies deutlich gemacht. TU9 hat von Anfang an das konsekutive, wissenschaftliche Bachelor/Master Studium als Standardmodell postuliert.

Ich aber behaupte: die größte Stärke des Diploms, nämlich die frühzeitige Vermittlung vertiefter wissenschaftlicher Grundlagen, wird sich im Bachelor nicht rekonstruieren lassen. Vielmehr gibt es einen Druck durch Politik und Akkreditierung, im Bachelor die wissenschaftlichen Grundlagen auszudünnen. Für dieses politisch gewollte Phänomen gibt es einen Begriff: Verfachhochschulung. Dieses Wort bitte ich rein technisch zu verstehen, denn niemand stellt den wichtigen Beitrag der Fachhochschulen in der praxisorientierten Ausbildung in Frage. Gerade in den technischen Fächern haben aber FH und Universität durchaus verschiedene Aufgaben.

\section{Verfachhochschulung als politisches Ziel}

An etlichen Uni-Fakultäten wird es durch Bologna jedoch eine Annäherung an FHs in Umfang und Tiefe der Studieninhalte geben. Sogar an großen Forschungsfakultäten wird die Akkreditierung einen gewissen Verfachhochschulungs-Druck erzeugen. Diese Entwicklung ist, ich sage es nochmals, politisch gewollt. Sie soll durch die Hintertür einen Defekt des deutschen Hochschulsystems beheben, dass nämlich 2/3 aller Studenten an Universitäten studieren und nur 1/3 an FHs, obwohl es eigentlich 
genau andersherum sein müsste. Eigentlich? - in den technischen Fachern eigentlich gar nicht, denn wir haben ohnehin viel zuwenig technische Absolventen auf Master- bzw. Diplom-Niveau.- Auch das sich abzeichnende Zweiklassensystem der Universitäten, nämlich eine Aufteilung in „Ausbildungsunis“ und „Forschungsunis“, ist politisch gewollt, aus demselben Grund. Das Instrument zur Implementierung des Zweiklassensystems ist natürlich die Exzellenzinitative.

Nun könnte man konstatieren, endlich ist Schluss mit der Lüge, dass alle Unis gleich sind, und Wettbewerb - auch mit den Fachhochschulen - belebt das Geschäft. Ich aber behaupte: die tendenzielle Verfachhochschulung des Bachelor wird an vielen Ausbildungsuniversitäten direkt auf den Master durchschlagen. Ein Grund ist, dass die Studentenströme sich am Zweiklassensystem orientieren werden, so dass die Ausbildungsunis kaum noch wissenschaftlich talentierte Studienanfänger bekommen, bzw. die guten Bachelor-Absolventen zum Master-Studium an eine Forschungsuni wechseln. Ein anderer Grund ist, dass ein verfachhochschulter Bachelor gar keinen wissenschaftlich anspruchsvollen Master mehr ermöglicht, weil die Voraussetzungen fehlen.

Mein zentraler Punkt ist deshalb folgende Behauptung: im Durchschnitt, also in der Fläche, wird das Niveau in den technischen Fächern sinken. Zwar werden die Talente an die Forschungs- oder „Elite"unis gehen, aber die Mehrheit der durchschnittlichen Studenten wird infolge der Verfachhochschulung in Bachelor und Master weniger lernen als vorher, und sie werden weniger Wissenschaftsbezug haben. Auch diese ,praxisorientierten“ Informatiker und Ingenieure werden einen guten Arbeitsmarkt vorfinden, wie ja auch BITKOM/GI deutlich machten.

Insgesamt ist aber das innovative Potential in den Ingenieurwissenschaften beschädigt. Das ist sehr gefährlich, denn wir brauchen mehr technische Master und nicht weniger, und wir brauchen sie mindestens auf dem Niveau des Diploms! Das durchschnittliche Niveau zu senken mit dem Argument, es gibt ohnehin nicht so viele Hochbegabte, und warum soll man die anderen mit Wissenschaft quälen, wenn doch gerade praxisorientierte Absolventen gute Aussichten am Arbeitsmarkt haben, übersieht: Gerade die ,praxisorientierten“ technischen Absolventen sind zukünftig noch mehr als heute - von Offshoring bedroht. Dies gilt besonders in der Informatik, und deshalb können wir nur mit Spitzenleistungen überleben. Im übrigen gilt: Theorie ohne Praxis ist steril, aber Praxis ohne Theorie ist unfruchtbar!

\section{Kompensationsmaßnahmen}

Trotzdem sind Bachelor/Master irreversibel. Man muss aus der Lage das Beste machen und Kompensationsmaßnahmen implementieren. Ich möchte zwischen politischen und technischen Kompensationsmaßnahmen unterscheiden.

Zunächst einmal muss man politisch konsequent Widerstand leisten gegen den quotierten MasterZugang. Wie auch schon das BITKOM/GI Papier feststellte, darf das einzige Zugangskriterium die fachliche Eignung der Bewerber sein. Denn wir haben sowieso zuwenig technische Absolventen auf Master- bzw. Diplom-Niveau. Deshalb ist es auch abwegig, Resourcen für die Masterausbildung zu limitieren bzw in den Bachelor umzulenken. Als nächstes muss man konsequent die Vermittlung wissenschaftlicher Grundlagen im technischen Bachelor auch an Ausbildungsunis forcieren. Nur so kann die Verfachhochschulung bekämpft und das Niveau des Master auf dem des Diplom gehalten werden. Ein zentrales Problem dabei sind die Akkreditierungsinstitutionen, da diese nicht von Universitätsvertretern dominiert werden und eine Tendenz bewiesen haben, nicht zu akkreditieren, wenn nach ihrer Meinung zuviel wissenschaftliche Grundlagen im Bachelor sind; ich erinnere nur an die verweigerte Akkreditierung der Kieler Informatik. Ich glaube deshalb, dass die GI, 4ING, aber auch GIBU immer noch zuwenig Einfluss auf die Akkreditierungsprozesse nehmen.

Ich möchte auch einige mehr technische Maßnahmen ansprechen. Dazu sollten wir uns vergegenwärtigen, dass die Abbrecherquoten im Bachelor keineswegs, wie von der Politik erhofft, 
gesunken sind, jedenfalls nicht in Informatik. Tatsächlich ist die Belastung im Studium für viele Studenten gestiegen. Ein Grund ist die verbindliche Vorgabe, dass ein ECTS-Punkt ca. 30

Arbeitsstunden zu entsprechen hat, was bei 60 ECTS pro Jahr 1800 Stunden ergibt, das sind 45 Wochen a 40 Stunden. Darin sind Vor- und Nachbereitung sowie Prüfungsvorbereitung enthalten. Verteilt man die 1800 Std aber nur auf die Vorlesungszeit, ergeben sich ca. 30 Wochen a 60 Stunden. Das ist völlig unzumutbar.

An vielen Fakultäten finden jedoch alle Prüfungen zu allen Veranstaltungen am Ende der Vorlesungsperiode statt, wodurch sich nicht nur eine Belastung ergibt, die viele scheitern lässt. Auch jede Nachhaltigkeit des Lernens wird zerstört, da man den Stoff nicht mehr in Ruhe ein zweites Mal bei der Prüfungsvorbereitung durcharbeiten kann (so wie früher bei der Vorbereitung aufs Vordiplom). Man muss deshalb die vorlesungsfreie Zeit für Prüfungsvorbereitung, Praktika, Tutorien und eben Prüfungen nutzen, um die Belastung stärker zu verteilen. Dies scheint mir offensichtlich, hat aber während meiner Passauer Dekanszeit erheblichen Widerstand generiert. Ich fand schließlich heraus, dass viele Studenten die vorlesungsfreie Zeit zum Jobben nutzen wollen. Das Bologna-Modell geht aber explizit von einem Vollzeitstudium aus und postuliert bei Nichteinhaltung der 30Std/ECTS Studienzeitverlängerungen. Man muss deshalb Klausurtermine entzerren, einen Teil der Klausuren ans Ende der vorlesungsfreien Zeit legen, und in der vorlesungsfreien Zeit zusätzliche Tutorien anbieten. Zusatztutorien wird man brauchen, um den „durchschnittlichen“ Studenten die wissenschaftlichen Grundlagen besser zu vermitteln. Dies muss nicht zu Lasten der Forschung gehen, da ja überall aus Studiengebühren zusätzliches Lehrpersonal eingestellt wird.

Man kann natürlich auch einfach die Anforderungen senken. Ich kenne Studiengänge, die die 30StdVorgabe glatt unterlaufen oder in Bachelor-Prüfungen jeden wissenschaftlichen Anspruch herausnehmen. Aber selbst wenn man am wissenschaftlichen Bachelor/Master festhält, wird das Ausbildungsniveau vielerorts unter Druck kommen. Um trotzdem das Niveau des Master wenn nicht für alle, so doch für viele zu halten, könnte man auf ein Instrument zurückgreifen, dass im angelsächsischen Raum schon lange bekannt ist: Honors Degrees, und damit verbunden, Zusatzangebote, die die wissenschaftlichen Grundlagen im Bachelor vertiefen. Damit kann man die Auswirkungen des Zweiklassensystems und der Verfachhochschulung lindern, und zumindest für einen Teil der Studenten das Niveau des Master auf der Höhe des alten Diploms halten. Allerdings hätte man so die TU9-Position, dass nämlich Bachelor/Master ein konsekutives, wissenschaftliches Studium darstellen, aufgegeben.

Schließlich sollte man auch darüber nachdenken, den Bachelor auf 7 Semester statt 6 anzulegen. Ich wage mir nämlich gar nicht auszudenken, was passiert, wenn das 8-jährige Gymnasium voll durchschlägt. Nur Politiker glauben, dass man in 8 Jahren soviel lernen kann wie in 9; deshalb muss man eigentlich, so wie in den USA, im 1. Bachelor-Semester fehlende Vorbildung kompensieren insbesondere in Mathematik und Naturwissenschaften. Einige Fakultäten haben das 7+3 Modell bereits eingeführt.

\section{Promotion und Nachwuchsförderung}

Auch auf die Chancen des wissenschaftlichen Nachwuches hat die gegenwärtige Umbruchsituation negative Auswirkungen. Denn die Politik stellt sich ja vor, dass eine Promotion in 3 Jahren abgeschlossen ist, und die jungen Talente im Anschluss sogleich eine Juniorprofessur übernehmen.

Aber das ist in den Ingenieurwissenschaften nur ausnahmsweise richtig. Eine ingenieurwissenschaftliche Promotion ist kein Durchlauferhitzer, in dem die Kandidaten in einem verschulten Modell zur Promotion ,geführt“ werden, und erst recht kein Instrument, wissenschaftliche Grundlagen nachzuholen, die im Bachelor oder gar Master fehlten. Eine ingenieurwissenschaftliche Promotion ist gemäß jahrzehntelanger Praxis in Deutschland und auch nach meiner Überzeugung eine substantielle, kreative und selbständige wissenschaftliche Leistung, die auf einem 
wissenschaftsorientierten Studium aufbaut. Wer versucht, den Ingenieurwissenschaften eine andere Promotionskultur überzustülpen, nimmt wiederum Substanz und Innovationspotential aus der Ausbildung heraus. Ein Grund dafür ist, dass Promotionen in Informatik oft Teil größerer Projekte sind, so dass man sie nur mit Gewalt in ein verschultes Raster zwängen kann.

Obendrein sehe ich das Risiko, dass in verschulten Modellen die Assistenten nicht mehr voll den Lehrstühlen zur Verfügung stehen (weil sie z.B. Kurse für „Schlüsselqualifikationen“ besuchen oder Zwischenberichte schreiben sollen), mit negativen Auswirkungen auf Projekte, aber auch auf die Lehre. Die TU9 hat ja zu dieser Problematik deutlich Stellung bezogen, nicht ohne Erfolg. Aber die Gefahr einer Verbolognisierung der Promotion ist für mich noch nicht gebannt.

Ein weiteres Problem des Nachwucheses hängt mit der Verfachhochschulung zusammen. Bereits jetzt kann man beobachten, dass manche Ausbildungsuniversitäten in Berufungsverfahren nicht mehr die besten Wissenschaftler auf die Berufungsliste setzen, sondern dass finanzielle Erwägungen und didaktische Breite Priorität gewinnen. Natürlich muss ein Professor auch lehren können. Jedoch deutet sich zur Zeit das Phänomen an, dass zwar die Top-Nachwuchsleute gute Positionen finden, kaum schlechter qualifizierte junge Forscher aber an den Ausbildungsunis nicht zum Zuge kommen (denn sie sind angeblich zu spezialisiert oder zu grundlagenorientiert). Viele dieser immer noch exzellent qualifizierten jungen Informatiker kehren deshalb der Wissenschaft den Rücken oder wandern in andere Länder aus, denn ihnen fehlt in Deutschland die Perspektive.

\section{Schluss}

In meinem Artikel „Paul Feyerabend und die Softwaretechnologie“ [2] habe ich mich über die sog. Konstruktivisten lustig gemacht, die glauben, dass es objektive Erkenntnis nicht gibt, sondern „wissenschaftliche Tatsachen“ nur soziale Konstrukte sind, die interessengesteuert entstehen. In der Tat geht ja - wie ich damals anmerkte - aus Newtons kürzlich aufgefundenem Geheimtestament hervor, dass er das Fallgesetz nur erfunden hat, um seine Lebensabschnittspartnerin am Höhenflug zu hindern.

Leider wird reale Politik aber oft nicht von objektiven Erwägungen getrieben, sondern von Konstrukten, wie z.B. der Vorstellung, dass das Bachelor/Master-System eine - wie auch immer geartete - Verbesserung der Ausbildung mit sich bringt. Der Bologna-Prozess orientiert sich jedoch am „durchschnittlichen“ Fach und am ,durchschnittlichen“ Studenten; er hat weder auf die besonderen Bedürfnisse technischer Fächer Rücksicht genommen, noch die besondere Situation technisch-wissenschaftlich Begabter respektiert. Es wird Zeit, dass die technischen Fächer zur Dekonstruktion schreiten und im Interesse der internationalen Konkurrenzfähigkeit Deutschlands Bachelor und Master so implementieren, dass das hohe Niveau der technischen Ausbildung erhalten bleibt.

\section{Literatur}

[1] „Bologna-Prozess für Qualität in der Hochschulbildung nutzen“. Presseinformation von BITKOM/GI/FTI/FTEI, Januar 2008.

[2] G. Snelting: Paul Feyerabend und die Softwaretechnologie. Informatik Spektrum, Oktober 1998, S. 273-276.

Prof. Dr.-Ing. Gregor Snelting war neun Jahre an der Universität Passau tätig, zuletzt als Dekan der Fakultät für Informatik und Mathematik. Zum April 2008 wechselte er an die Universität Karlsruhe. Prof. Snelting wurde im März 2008 zum neuen Vorsitzenden des GIBU gewählt. 
